\title{
The importance of mixed methods How validation and voice may interact effectively \\ Christopher Charles Deneen
}

\begin{abstract}
The use of qualitative and quantitative research methods, together (henceforth referred to as mixed methods) is increasing in popularity within social science research. Mixed methods approaches have tremendous potential to provide a more complete picture of the topic under study and enhance validation of findings. Mixed methods may provide balanced results that allow for highly defensible interpretations while allowing the 'voice' of participants to form compelling narratives. Significant challenges exist, however in proper implementation.

This paper explores the potential and challenges of conducting mixed methods in the social sciences through focusing on three mixed-methods studies conducted by the author. Mixed methods data collection, analysis and presentation of findings are addressed. Research was conducted in the field of education studies, but implications are made clear for the broader field of social sciences. Key topics include developing complimentary relationships between quantitative surveys and interviews, integrating MANOVA and SEM with qualitative coding, and reporting mixed findings as an integrated whole. Difficulties and challenges at different stages of mixed methods implementation are addressed as well. The paper concludes by discussing implications for research directions, researcher practice and professional development of academic staff.
\end{abstract}

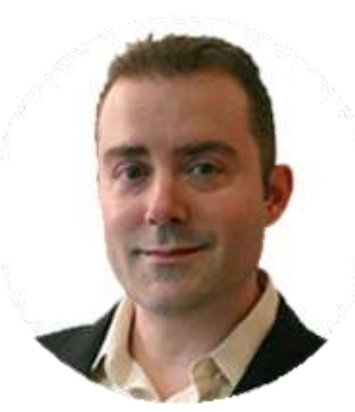

Dr. Christopher Charles Deneen is an assistant professor with the National Institute of Education at Nanyang Technological University. His research focuses on using mixed methods to explore higher education assessment and evaluation, with an emphasis on how these interact with learning, leadership, technology, and change management. 